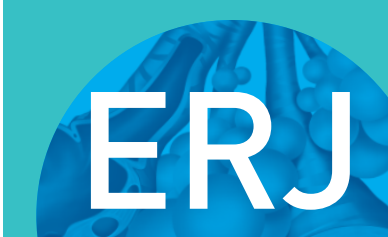

open research

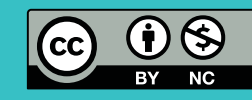

\section{Let research leave you breathless, not physical exercise!}

\author{
Matteo Bonini and Omar S. Usmani \\ National Heart and Lung Institute (NHLI), Imperial College London \& Royal Brompton Hospital, London, UK.
}

Correspondence: Matteo Bonini, Airways Disease Section, National Heart and Lung Institute (NHLI), Imperial College London \& Royal Brompton Hospital, Dovehouse Street, London SW3 6LY, UK.

E-mail: m.boninidimperial.ac.uk

$@$ ERSpublications

Proper endotyping of EIB and precision medicine strategies would allow subjects to fully profit from the very beneficial effects of exercise, without incurring health risks or affecting performances http://ow.ly/spjT30irzjX

Cite this article as: Bonini M, Usmani OS. Let research leave you breathless, not physical exercise!. ERJ Open Res 2018; 4: 00010-2018 [https://doi.org/10.1183/23120541.00010-2018].

Regular physical activity is strongly recommended by healthcare systems worldwide and evidence-based guidelines and is one of the most effective approaches for preventing chronic inflammatory diseases and maintaining health status [1]. Indeed, extensive evidence exists on the beneficial effect of physical training and rehabilitation programmes in asthma [2]. Physical activity has been shown to improve quality of life, exercise capacity, pulmonary function and symptoms, as well as reduce airway inflammation and bronchial responsiveness in patients with asthma [3-5]. However, intense physical exercise may trigger airway narrowing by imposing a high demand on the respiratory system, requiring subjects to ventilate primarily through the mouth and by-pass the nasal filter, with a subsequent increased pulmonary exposure to inhalant allergens, pollutants, irritants and adverse (i.e. cold, dry) environmental conditions [6]. Such airway narrowing, which transiently occurs as a result of exercise, is defined as exercise-induced bronchoconstriction (EIB) [7]. Interestingly, in a 5-year prospective study, subjects who stopped training experienced an attenuation, or in some circumstances disappearance, of EIB, whereas bronchial responsiveness, exercise-induced respiratory symptoms and eosinophilic airway inflammation increased amongst those who continued strenuous physical exercise, regardless of the pharmacological treatment strategies [8]. Put into context, ongoing intense training appears to be a causative, and not just a concomitant, factor of airway inflammation and narrowing.

The prevalence of EIB ranges from $5 \%$ to $20 \%$ in the general population. EIB has also been reported to occur in almost all asthmatic subjects, reflecting the level of disease control, with EIB occurring more frequently in more severe and uncontrolled patients [9]. Furthermore, EIB is particularly frequent, even in the absence of underlying clinical asthma, in athletes [10], children [11] and subjects with rhinitis [12]. The intensity, duration and type of training has been also associated with the occurrence of airway hyperresponsiveness and symptoms, with EIB being more prevalent in subjects practicing swimming, endurance and winter sport disciplines [7]. EIB typically develops within $15 \mathrm{~min}$ following at least $5-8 \mathrm{~min}$ of high intensity ( $>85 \%$ of maximal voluntary ventilation) aerobic training, and spontaneously

Received: Jan 222018 | Accepted: Jan 272018

Conflict of interest: O.S. Usmani has received fees for consultancy from AstraZeneca, Boehringer Ingelheim, Chiesi, GlaxoSmithKline, NAPP, Mundipharma, Sandoz, Cipla, Takeda, Zentiva and Trundell Medical. He has received grants from AstraZeneca, Boehringer Ingelheim, Chiesi, Prosonix and Edmond Pharma. He has also received speakers fees from Boehringer Ingelheim, Chiesi, Cipla, NAPP, Mundipharma, Sandoz and Aerocrine.

Support statement: This work has been supported by a RESPIRE2 ERS/Marie-Curie Fellowship awarded to M. Bonini to develop the project "Inflammatory and functional biomarkers of the Th2-low endotype in exercise-induced asthma". Funding information for this article has been deposited with the Crossref Funder Registry.

Copyright $\odot$ ERS 2018. This article is open access and distributed under the terms of the Creative Commons Attribution Non-Commercial Licence 4.0. 
resolves within $60 \mathrm{~min}$ [7]. After an episode of EIB, there is often a refractory period of about $1-3 \mathrm{~h}$ during which, if exercise is repeated, the bronchoconstriction is less accentuated [7]. Common symptoms include cough, dyspnoea, breathlessness, wheezing and chest tightness [7].

A careful medical history and physical examination is always recommended for diagnosing EIB [13]. The use of specific questionnaires for screening allergic and respiratory diseases in exercisers and athletes represent an additional valuable and accessible diagnostic tool [14]. However, research has consistently shown a poor relationship between the presence of asthma-like symptoms and objective evidence of EIB [15]. Furthermore, pulmonary function tests at baseline seem to be poorly predictive of EIB in people engaging in regular physical activity, often being within the normal values even in the presence of disease [16]. Thus, in order to establish a secure diagnosis of EIB, it is critical to perform objective testing to confirm dynamic changes in airway calibre and function [7]. It is still a matter of ongoing debate as to which bronchial provocation challenge should be preferred, and whether a single test could be enough to set a definite diagnosis [17].

The increase in airways osmolarity due to respiratory water loss and the vasodilation associated with airways rewarming have been commonly reported to be the major determinants of EIB (osmotic and thermal theories) [18, 19]. However, as with the current approach in asthma phenotypes, different endotypes (i.e. disease subtypes specifically defined by functional or pathological molecular mechanisms and/or treatment response) of EIB have been recently reported (figure 1) [20]. Intense physical training may induce a transient status of immune downregulation with a shift towards a relatively prevalent T2-high response, clinically associated with an increased prevalence of atopy [10, 21]. However, despite in a large proportion of EIB subjects the occurrence of bronchial obstruction following exercise is associated with allergic diseases [10] and markers of a T2-high response [22]. EIB is also present in subjects with no

Major postulated mechanisms

Recently suggested mechanisms

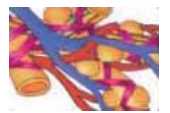

Thermal mechanism
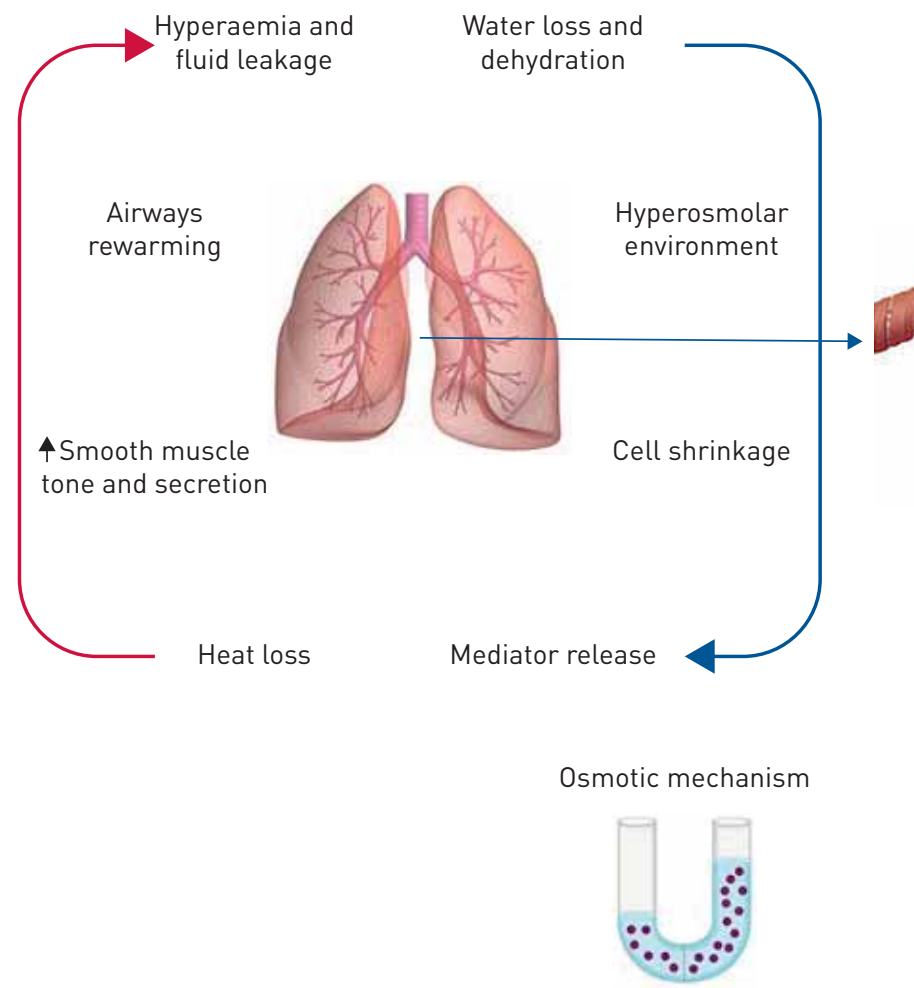

Autonomic dysregulation

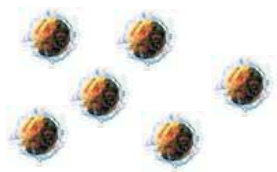

Enhanced T2-high response
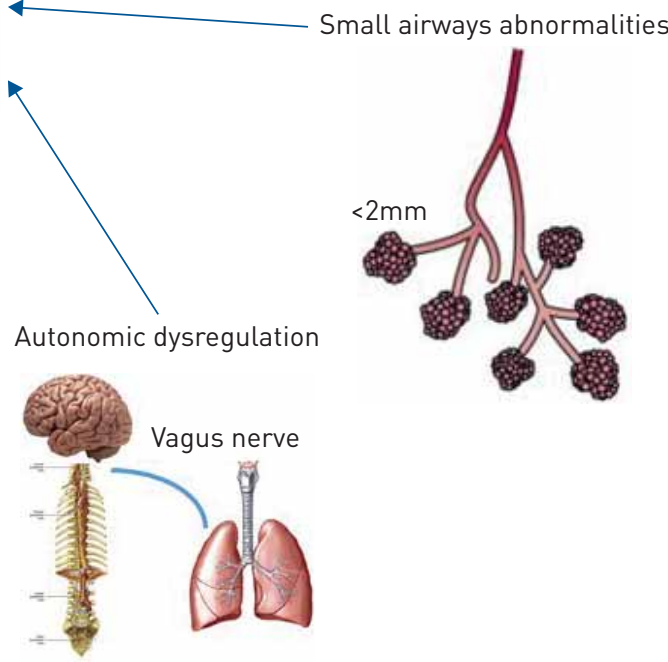

FIGURE 1 Pathophysiological mechanisms of exercise-induced bronchoconstriction (EIB). 
evidence of atopic sensitisation. In such non-T2-high variants of EIB, the bronchial epithelial damage directly caused by physical activity has been suggested as a relevant pathogenic mechanism [20]. A direct injury of the bronchial epithelium might also be caused by viral upper respiratory tract infections, which are reported to occur more frequently in athletes [23]. Inflammatory mediators released by the damaged epithelium have been found both in sputum (i.e. interleukin-8) [24] and in serum (i.e. nerve growth factor) [25] and seem to be associated with a neutrophilic or mixed neutrophilic/eosinophilic inflammatory response. Furthermore, the evidence that CC16 proteins, secreted by Club Cells in the distal bronchioles to protect the respiratory tract against oxidative stress and inflammation, are increased in urine and serum following an exercise challenge [26], has prompted renewed and increasing interest in the role of small airways in EIB. Notably, small airway abnormalities have been related to the onset and severity of asthma and may occur even in the absence of a response in the large airways [27]. In addition, an alternative non-inflammatory mechanism, autonomic dysregulation with an enhanced parasympathetic response, measured by both pupillometry and heart rate variability, has been shown to significantly relate to EIB [28].

Despite the above reported research findings, no clear classification of EIB endotypes have yet been adopted in clinical practice and the distinction between T2-high and non-T2-high variants is currently made only by exclusion of a T2-high signature. Therefore, the lack of specific functional and inflammatory biomarkers to classify T2-low EIB subjects currently prevents clear identification of these endotypes and therefore, an adequate design of clinical research trials. Proper endotyping of EIB would also have extremely relevant clinical translational impact, by significantly contributing to improved disease assessment and management. In fact, although prevention and treatment of EIB is mainly based on the effective use of short- and long-acting $\beta_{2}$-agonists, high heterogeneity in individual therapeutic responses, as well as the occurrence of tolerance and side-effects $[29,30]$, have been observed, suggesting the existence of subpopulations requiring an endotype-driven approach to optimise therapy. Moreover, while several targeted therapies are available or under development for a "precision medicine" in T2-high asthma, no tailored strategies are currently available for the non-T2-high forms, in which corticosteroids have shown to be poorly effective. This could be also of relevance in professional athletes in view of the World Anti-Doping Agency regulations (www.wada-ama.org).

It can be appreciated that further research is desirable following these interesting and promising preliminary findings in order to fully address the unmet needs as outlined above, and to allow the vast population of subjects undertaking physical activity to fully profit from the very beneficial effects of exercise, without incurring health risks or affecting their performance and quality of life.

\section{References}

1 Latimer-Cheung AE, Toll BA, Salovey P. Promoting increased physical activity and reduced inactivity. Lancet 2013; 381: 114 .

2 Carson KV, Chandratilleke MG, Picot J, et al. Physical training for asthma. Cochrane Database Syst Rev 2013; 9: CD001116.

3 Moreira A, Delgado L, Haahtela T, et al. Physical training does not increase allergic inflammation in asthmatic children. Eur Respir J 2008; 32: 1570-1575.

4 Eichenberger PA, Diener SN, Kofmehl R, et al. Effects of exercise training on airway hyperreactivity in asthma: a systematic review and meta-analysis. Sports Med 2013; 43: 1157-1170.

5 Del Giacco SR, Garcia-Larsen V. Aerobic exercise training reduces bronchial hyper-responsiveness and serum pro-inflammatory cytokines in patients with asthma. Evid Based Med 2016; 21: 70.

6 Bonini M, Palange P. Exercise-induced bronchoconstriction: new evidence in pathogenesis, diagnosis and treatment. Asthma Res Pract 2015; 1: 2.

7 Parsons JP, Hallstrand TS, Mastronarde JG, et al. An official American Thoracic Society clinical practice guideline: exercise-induced bronchoconstriction. Am J Respir Crit Care Med 2013; 187: 1016-1027.

8 Helenius IJ, Rytilä P, Sarna S, et al. Effect of continuing or finishing high-level sports on airway inflammation, bronchial hyperresponsiveness, and asthma: a 5-year prospective follow-up study of 42 highly trained swimmers. J Allergy Clin Immunol 2002; 109: 962-968.

9 Smoliga JM, Weiss P, Rundell KW. Exercise induced bronchoconstriction in adults: evidence based diagnosis and management. BMJ 2016; 352: h6951.

10 Bonini M, Gramiccioni C, Fioretti D, et al. Asthma, allergy and the Olympics: a 12-year survey in elite athletes. Curr Opin Allergy Clin Immunol 2015; 15: 184-192.

11 Randolph C. Exercise-induced bronchospasm in children. Clin Rev Allergy Immunol 2008; 34: 205-216.

12 Bousquet J, Van Cauwenberge P, Khaltaev N, et al. Allergic rhinitis and its impact on asthma. J Allergy Clin Immunol 2001; 108: Suppl 5, S147-S334.

13 Price OJ, Hull JH, Ansley L, et al. Exercise-induced bronchoconstriction in athletes - a qualitative assessment of symptom perception. Respir Med 2016; 120: 36-43.

14 Bonini M, Braido F, Baiardini I, et al. AQUA: Allergy Questionnaire for Athletes. Development and validation. Med Sci Sports Exerc 2009; 41: 1034-1041.

15 Ansley L, Kippelen P, Dickinson J, et al. Misdiagnosis of exercise-induced bronchoconstriction in professional soccer players. Allergy 2012; 67: 390-395.

16 Bonini M, Lapucci G, Petrelli G, et al. Predictive value of allergy and pulmonary function tests for the diagnosis of asthma in elite athletes. Allergy 2007; 62: 1166-1170. 
17 Hull JH, Ansley L, Price OJ, et al. Eucapnic voluntary hyperpnea: gold standard for diagnosing exercise-induced bronchoconstriction in athletes? Sports Med 2016; 46: 1083-1093.

18 Anderson SD, Daviskas E. The mechanism of exercise-induced asthma is .... J Allergy Clin Immunol 2000; 106: 453-459.

19 McFadden ER. Hypothesis: exercise-induced asthma as a vascular phenomenon. Lancet 1990; 1: 880-883.

20 Couto M, Kurowski M, Moreira A, et al. Mechanisms of exercise-induced bronchoconstriction in athletes: current perspectives and future challenges. Allergy 2018; 73: 8-16.

21 Lakier Smith L. Overtraining, excessive exercise, and altered immunity: is this a $\mathrm{T}$ helper-1 versus $\mathrm{T}$ helper-2 lymphocyte response? Sports Med 2003; 33: 347.

22 Kurowski M, Jurczyk J, Jarzębska M, et al. Serum but not exhaled breath condensate periostin level is increased in competitive athletes. Clin Respir J 2018 in press [https://doi.org/10.1111/crj.12759].

23 Gleeson M, Pyne DB. Respiratory inflammation and infections in high-performance athletes. Immunol Cell Biol 2016; 94: 124-131.

24 Chimenti L, Morici G, Paternò A, et al. Bronchial epithelial damage after a half-marathon in non-asthmatic amateur runners. Am J Physiol Lung Cell Mol Physiol 2010; 298: L857-L862.

25 Bonini M, Fioretti D, Sargentini V, et al. Increased nerve growth factor serum levels in top athletes. Clin J Sport Med 2013; 23: 228-231.

26 Tufvesson E, Svensson H, Ankerst J, et al. Increase of club cell (Clara) protein (CC16) in plasma and urine after exercise challenge in asthmatics and healthy controls, and correlations to exhaled breath temperature and exhaled nitric oxide. Respir Med 2013; 107: 1675-1681.

27 Bonini M, Usmani OS. The role of the small airways in the pathophysiology of asthma and chronic obstructive pulmonary disease. Ther Adv Respir Dis 2015; 9: 281-293.

28 Stang J, Stensrud T, Mowinckel P, et al. Parasympathetic activity and bronchial hyperresponsiveness in athletes. Med Sci Sports Exerc 2016; 48: 2100-2107.

29 Bonini M, Permaul P, Kulkarni T, et al. Loss of salmeterol bronchoprotection against exercise in relation to ADRB2 Arg16Gly polymorphism and exhaled nitric oxide. Am J Respir Crit Care Med 2013; 188: 1407-1412.

30 Bonini M, Di Mambro C, Calderon MA, et al. Beta ${ }_{2}$-agonists for exercise-induced asthma. Cochrane Database Syst Rev 2013; 10: CD003564. 